Maria Cláudia Dantas Porfirio Borges ANDRÉ 1 Patrícia Pimentel SANTOS ${ }^{1}$ Maria Raquel Hidalgo CAMPOS $^{2}$

Liana Jayme BORGES ${ }^{2}$

Álvaro Bisol SERAFINI ${ }^{1}$

Correspondência para:

MARIA CLÁUDIA DANTAS PORFIRIO

BORGES ANDRÉ

Rua 01, n $967-$ apt $^{\circ} 1301$ - Ed. Ilhas

Christmas-Setor Oeste

74115-040 - Goiânia-GO

mcdantas@brturbo.com.br

Recebido para publicação: 13/07/2005 Aprovado para publicação: 10/12/2006

\title{
Utilização do Antibiograma como Ferramenta de Tipagem Fenotípica de Staphylococcus aureus Isolados de Manipuladores, Leite Cru e Queijo Minas Frescal em Laticínio de Goiás, Brasil
}

1 - Instituto de Patologia Tropical e Saúde Pública/UFG.

2 - Faculdade de Nutrição/UFG

\section{Resumo}

O trabalho teve por objetivo isolar, identificar e caracterizar fenotipicamente utilizando o antibiograma, Staphylococcus aureus isolados de manipuladores, leite cru e queijo Minas Frescal, em um laticínio de Goiás. Durante doze meses (Março/2004 a Fevereiro/ 2005) 140 amostras foram analisadas. As cepas foram isoladas de $75 \%$ dos manipuladores resultando em 31 (33,7\%) isolados a partir de 92 amostras coletadas (46 de mãos e 46 de nasofaringe). Das 24 amostras de leite cru, $18(75,0 \%)$ foram positivas, resultando em 26 isolados, com média de contagem de $1,1 \times 10^{5} \mathrm{UFC} / \mathrm{ml}$. Das 24 amostras de queijo 17 (70,8\%) foram positivas originando 20 isolados, com média de 3,8 x 104 UFC/g, sendo 13 (54,2\%) com população acima do limite estabelecido pela legislação brasileira $\left(10^{3} \mathrm{UFC} / \mathrm{g}\right)$. A tipagem das cepas foi feita pelo antibiograma, através do método de difusão em placas. Todos os isolados foram susceptíveis à ciprofloxacina e gentamicina. Foi observada resistência em cinco (6,5\%) isolados para eritromicina, 19 (24,7\%) para tetraciclina, um (1,3\%) para vancomicina, quatro $(5,2 \%)$ para oxacilina e $53(68,8 \%)$ para penicilina. Onze $(14,3 \%)$ isolados foram susceptíveis a todos os antibióticos testados e 18 (23,4\%) foram resistentes a mais de um antibiótico. $\mathrm{O}$ antibiograma permitiu a classificação das cepas em doze perfis diferentes (A-L), porém não foi uma técnica eficiente em determinar a origem da contaminação final do queijo.

\section{Introdução}

Os produtos lácteos são freqüentemente envolvidos em surtos de toxinfecções alimentares em todo o mundo. Muitos microrganismos patogênicos podem ser veiculados ao homem através do consumo de leite e seus derivados, entre eles a bactéria Staphylococcus aureus ${ }^{1,2,3}$.

O homem e os animais são os principais reservatórios de $S$. aureus. A contaminação de alimentos com $S$. aureus deve-se primariamente à sua presença na matéria-prima ${ }^{2}$, apesar de um grande número de surtos de toxinfecções alimentares envolverem cepas de origem humana como fontes de contaminação ${ }^{4}$. A cavidade nasal é o principal habitat dos estafilococos no homem e sua presença em manipuladores assintomáticos está bem documentada $5,6,7,8,9,10,11,12,13$ e contribui para a contaminação de produtos lácteos. Os $S$. aureus são considerados os mais importantes na epidemiologia das mastites em vacas de leite e o patógeno mais abundante em leite $\mathrm{cru}^{14,15,16,17,18}$. A mastite bovina é uma doença epidêmica de difícil tratamento e controle, sendo a causa mais freqüente do uso de antimicrobianos em propriedades leiteiras ${ }^{17,19}$. $\mathrm{O}$ uso indiscriminado de antibióticos em alimentos, seja para fins terapêuticos, profilaticamente, ou ainda, incorporados à alimentação como suplemento dietético, facilita a disseminação de microrganismos 
designados MDR (multidroga resistentes). Entre os problemas ocasionados por estes resíduos, destaca-se a seleção de cepas resistentes, como também, a possibilidade de transferência de resistência entre espécies, através de mecanismos genéticos ${ }^{20}$.

Sob condições favoráveis, estas bactérias podem proliferar e constituir risco à Saúde Pública pela sua capacidade de produzir enterotoxinas. A ingestão de alimentos contendo enterotoxinas préformadas é responsável pela intoxicação alimentar estafilocócica, que é uma das doenças de origem alimentar mais comuns em vários países do mundo ${ }^{2,21}$.

O queijo Minas Frescal é um queijo mole, de alta umidade, consumido fresco ${ }^{22}$. Fatores que influenciam a qualidade do queijo são: a contaminação da matéria-prima (leite) e demais ingredientes, a contaminação a partir de manipuladores, equipamentos e após o processamento industrial e o tempo de exposição a temperaturas que favoreçam a proliferação de microrganismos ${ }^{23}$.

A tipagem de $S$. aureus tem sido usada para sugerir a origem desta bactéria em alimentos ${ }^{4,9,24}$. O antibiograma tem sido um método útil de tipagem bacteriana, contribuindo na investigação de fontes de contaminação, sendo a técnica amplamente disponível e padronizada e podendo ser utilizada com várias espécies microbianas ${ }^{25}$. Sua principal desvantagem consiste na variabilidade da expressão da resistência, a instabilidade devido à transmissão horizontal e perda dos elementos genéticos extracromossômicos ${ }^{26}$.

O objetivo deste trabalho foi caracterizar fenotipicamente, isolados de $S$. aureus obtidos de queijo Minas Frescal, leite cru e manipuladores de um laticínio, utilizando o antibiograma e determinar a similaridade, se presente, entre os isolados do queijo e suas possíveis fontes de contaminação.

\section{Material e Métodos}

No período de Março de 2004 a
Fevereiro de 2005 foram coletadas 140 amostras em um laticínio, no estado de Goiás: 24 amostras de leite cru, 24 de queijo ( \pm $500 \mathrm{~g})$ e 46 amostras de mãos e 46 de nariz a partir de quatro manipuladores, funcionários da área de processamento de queijo. As análises foram realizadas no Laboratório de Microbiologia de Alimentos e de Ambientes da Universidade Federal de Goiás.

A contagem de $S$. aureus para as amostras de leite cru e queijo Minas frescal foi realizada no Ágar Baird-Parker ${ }^{27}$ e o isolamento de nasofaringe e mãos, após os suabes terem sido previamente inoculados em BHI, foi a partir do ágar Manitol Salgado ${ }^{8}$. Para a identificação utilizou-se: Coloração de Gram, produção de catalase, coagulase, termonuclease e acetoína (VP), fermentação anaeróbica da glicose e manitol ${ }^{27,28}$. A tipagem fenotípica foi realizada por antibiograma, através da técnica de difusão em placas $^{29}$. Foram testados os seguintes discos de antimicrobianos: eritromicina, ciprofloxacina, tetraciclina, gentamicina, vancomicina, oxacilina e penicilina. Uma cepa padrão de Staphylococcus aureus (ATCC 25923) foi usada como controle.

\section{Resultados e Discussão}

Das 140 amostras de leite cru, queijo Minas Frescal e manipuladores coletadas, 66 $(47,1 \%)$ foram positivas para Staphylococcus aureus e destas foram obtidos 77 isolados (Tabela 1).

Dos quatro manipuladores, três $(75 \%)$ apresentaram-se contaminados pelo menos duas vezes nas mãos e/ou nariz durante todo o período da coleta. Das 92 amostras coletadas neste período, 31 (33,7\%) foram positivas para $S$. aureus sendo $16(34,8 \%)$ isolados obtidos a partir de 46 suabes de nasofaringe e $15(32,6 \%)$ isolados a partir de suabes de mãos (Tabela 1). As amostras foram coletadas em dias diferentes e os isolados foram comparados através do antibiograma. Esses resultados mostram porcentagens de contaminação superiores 
Tabela 1 - Contaminação de S. aureus em manipuladores, leite cru e queijo Minas Frescal em um laticínio de Goiás, Brasil

\begin{tabular}{|c|c|c|c|c|c|}
\hline $\begin{array}{c}\text { Fontes }\left(\mathrm{N}^{\circ} . \text { de }\right. \\
\text { amostras coletadas) }\end{array}$ & \multicolumn{2}{|c|}{$\begin{array}{c}\text { Amostras positivas } \mathrm{N}^{\circ} . \\
(\%)\end{array}$} & \multicolumn{2}{|c|}{$\mathrm{N}^{\circ}$ isolados } & $\begin{array}{l}\text { Média (contagens mínimas e } \\
\text { máximas) UFC/ml ou g }\end{array}$ \\
\hline Nariz (46) & \multirow{2}{*}{$31(33,7)$} & $16(34,8)$ & \multirow{2}{*}{31} & 16 & ND \\
\hline Mãos(46) & & $15(32,6)$ & & 15 & ND \\
\hline Leite cru (24) & \multicolumn{2}{|c|}{$18(75,0)$} & \multicolumn{2}{|c|}{26} & $1,1 \times 10^{5}\left(<1,0 \times 10^{1}\right.$ a $\left.7,5 \times 10^{5}\right)$ \\
\hline Queijo(24) & \multicolumn{2}{|c|}{$17(70,8)$} & \multicolumn{2}{|c|}{20} & $3,8 \times 10^{4}\left(<1,0 \times 10^{1}\right.$ a $\left.3,4 \times 10^{5}\right)$ \\
\hline Total $(140)$ & \multicolumn{2}{|c|}{$66(47,1)$} & \multicolumn{2}{|c|}{77} & - \\
\hline
\end{tabular}

Tabela 2 - Susceptibilidade antimicrobiana de S. aureus isolados de manipuladores, leite cru e queijo Minas Frescal em um laticínio de Goiás, Brasil

\begin{tabular}{l|c|ccccccc}
\hline \multirow{2}{*}{$\begin{array}{c}\text { Amostras } \\
\text { Positivas }\end{array}$} & \multirow{2}{*}{$\begin{array}{c}\text { N }^{0} \text {. de } \\
\text { isolado }\end{array}$} & \multicolumn{7}{|c}{ Resistência a No $(\%)$} \\
\cline { 5 - 8 } & $\mathrm{S}$ & ERI & CIP & TET & GEN & VAN & OXA & PEN \\
\hline Manipuladores (31) & 31 & $2(6,4)$ & $0(0)$ & $5(16.1)$ & $0(0)$ & $1(3,2)$ & $3(9.7)$ & $23(74.2)$ \\
Leite cru (18) & 26 & $2(7,7)$ & $0(0)$ & $9(34,6)$ & $0(0)$ & $0(0)$ & $0(0)$ & $18(69.2)$ \\
Queijo (17) & 20 & $1(5,0)$ & $0(0)$ & $5(25,0)$ & $0(0)$ & $0(0)$ & $1(5,0)$ & $12(60.0)$ \\
Total (66) & 77 & $5(6,5)$ & $0(0)$ & $19(24.7)$ & $0(0)$ & $1(1.3)$ & $4(5.2)$ & $53(68.8)$ \\
\hline
\end{tabular}

ERI: eritromicina, CIP: ciprofloxacina, TET: tetraciclina, GEN: gentamicina, VAN: vancomicina, OXA: oxacilina, PEN: penicilina

Tabela 3 - Perfis de susceptibilidade antimicrobiana originados de cepas de $S$. aureus isoladas de um laticínio de Goiás, Brasil

\begin{tabular}{|c|c|c|}
\hline Número da amostra e origem ${ }^{a}$ & Perfil de susceptibilidade ${ }^{b}$ & Fenótipo \\
\hline $\begin{array}{l}\text { 2,4M4, 11M1, 12M2, 8LT, 17LT, 18LT, 5QT } \\
\text { 19,QT, 22QT }\end{array}$ & SSSSSSS & A \\
\hline $\begin{array}{l}6 \mathrm{M} 2,4 \mathrm{~N} 4,10 \mathrm{M} 4,12 \mathrm{M} 1,5 \mathrm{~L}, 14 \mathrm{~L}, 16 \mathrm{~L}, 15 \mathrm{Q}, 18 \mathrm{Q} \\
21 \mathrm{Q}\end{array}$ & ISSSSSS & B \\
\hline $\begin{array}{l}8,12,14,20 \mathrm{~N} 4,8 \mathrm{M} 4,13,15 \mathrm{M} 1,8 \mathrm{~L}^{\prime}, 9 \mathrm{~L}^{\prime}, 10 \mathrm{~L}^{\prime}, 12 \mathrm{~L}, 12^{\prime}, \\
17 \mathrm{~L}^{\prime}, 19 \mathrm{~L}^{\prime}, 20 \mathrm{~L}, 20 \mathrm{~L}^{\prime}, 22 \mathrm{~L}, 4 \mathrm{Q}^{\prime}, 7 \mathrm{Q}, 8 \mathrm{Q}, 16 \mathrm{Q}^{\prime}, 23 \mathrm{Q}\end{array}$ & SSSSSSR & $\mathrm{C}$ \\
\hline $\begin{array}{l}1 \mathrm{~N} 2,3,5,7,10,15,17 \mathrm{~N} 4,15 \mathrm{M} 4,6,16 \mathrm{~N} 1,13 \mathrm{~L}^{\prime}, 16 \mathrm{~L}^{\prime} \\
6 \mathrm{Q}, 18 \mathrm{Q}^{\prime}\end{array}$ & ISSSSSR & $\mathrm{D}$ \\
\hline $13 \mathrm{~L}, 14 \mathrm{~L}^{\prime}, 3 \mathrm{Q}$ & ISRSSSS & E \\
\hline $5 \mathrm{M} 4,19 \mathrm{~N} 2,6 \mathrm{~L}, 6 \mathrm{~L}^{\prime}, 11 \mathrm{~L}, 4 \mathrm{Q}, 10 \mathrm{Q}, 24 \mathrm{Q}$ & SSRSSSR & $\mathrm{F}$ \\
\hline $13 \mathrm{Q}$ & RSSSSSR & G \\
\hline $11 \mathrm{~L}^{\prime}, 24 \mathrm{~L}$ & ISRSSSR & $\mathrm{H}$ \\
\hline $10 \mathrm{~N} 2,4 \mathrm{~L}, 15 \mathrm{~L}^{\prime}$ & RSRSSSR & $\mathrm{I}$ \\
\hline $16 \mathrm{M} 1,2 \mathrm{Q}$ & ISRSSRR & $\mathrm{J}$ \\
\hline $1 \mathrm{M} 1$ & RSSSRRR & K \\
\hline $19 M 2$ & ISRISRR & $\mathrm{L}$ \\
\hline
\end{tabular}

${ }^{\mathrm{a}} \mathrm{M}$-Mão; N-Nariz; L-Leite; Q-Queijo.

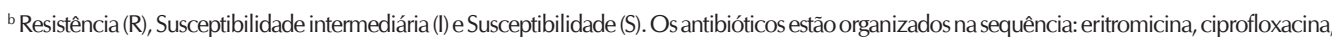
tetraciclina, gentamicina, vancomicina, oxacilina e penicilina. 
aos obtidos em outros países.

No Kuwait, 26,6\% dos trabalhadores de um restaurante portavam $S$. aureus na nasofaringe ${ }^{5}$; no Chile, $65,5 \%$ dos manipuladores de alimentos apresentaram a bactéria na nasofaringe, orofaringe, mãos e unhas ${ }^{31}$. Na Turquia, $70 \%$ dos manipuladores estavam com as mãos contaminadas?. No Brasil, estudos demonstram a presença da bactéria em manipuladores em Teresina, PI, com prevalência variando de $43,3 \%$ a $49,5 \%{ }^{7}$; em Porto Alegre, RS, de $30 \%$ a $35,2 \%{ }^{9,12}$. O homem é considerado portador de $S$. aureus na nasofaringe em $37,2 \%$ da população em geral $^{30}$.

Das 24 amostras de leite cru coletadas, $18(75,0 \%)$ apresentaram S. aureus, produzindo 26 isolados. Resultados elevados também foram encontrados em Trinidad ${ }^{31}$, onde $97,6 \%$ das amostras apresentavam $S$. aureus. A média de contagem no leite cru foi de $1,1 \times 10^{5} \mathrm{UFC} / \mathrm{mL}$ (Tabela 1). Isto assume grande importância em Saúde Pública, considerando que as enterotoxinas podem ser detectadas com inóculos de $10^{3}$ $\mathrm{UFC} / \mathrm{g}^{10}$. S. aureus é a bactéria mais freqüentemente isolada de mastite bovina em todo o mundo ${ }^{16,32}$ e aproximadamente em $20 \%$ das amostras de leite de vacas com mastite no Brasil ${ }^{23}$. Considerando que o leite foi coletado no tanque de recepção e demonstrou altas contagens, cepas patogênicas podem disseminar para o consumidor a partir da fabricação de derivados do leite, principalmente se este não sofrer processamento adequado.

Cepas de $S$. aureus foram encontradas em $17(70,8 \%)$ das 24 amostras de queijo Minas Frescal coletadas, obtendo-se 20 isolados. A contagem da bactéria variou de $<1,0 \times 10^{1}$ a $3,4 \times 10^{5}$, com média de $3,8 \times$ $10^{4} \mathrm{UFC} / \mathrm{g}$ (Tab. 1). Treze amostras $(54,2 \%)$ estavam acima do limite $\left(10^{3} \mathrm{UFC} / \mathrm{g}\right)$ estabelecido pela legislação vigente no Brasil $^{11}$. Na França, um estudo durante o período de 1988 a 1997 demonstrou que o leite e derivados foram responsáveis por $59 \%$ dos surtos de toxinfecção alimentar e que o $S$. aureus foi o patógeno mais freqüentemente associado a surtos devidos ao consumo de queijos fabricados a partir de leite cru ou desconhecido ${ }^{2}$. No Brasil estudos demonstram uma porcentagem maior de contaminação do queijo. Em Blumenau, SC, 95\% das amostras de queijo continham $S$. aureus ${ }^{34}$, em Poços de Caldas, MG, 50\% das amostras, com contagens de $10^{5} \mathrm{UFC} / \mathrm{g}$ em média ${ }^{1}$. Em Cuiabá, MT, $96,7 \%$ das amostras de queijo estavam fora dos padrões microbiológicos nacionais ${ }^{23} \mathrm{e}$ no Rio de Janeiro, RJ, 77\% das amostras estavam contaminadas e $17,7 \%$ delas com contagens acima do limite estabelecido ${ }^{22}$.

Dos 77 isolados, 53 (68,8\%) foram resistentes à penicilina, $19(24,7 \%)$ à tetraciclina, cinco $(6,5 \%)$ resistentes à eritromicina, quatro $(5,2 \%)$ à oxacilina e um $(1,3 \%)$ à vancomicina (Tabela 2$)$. Onze $(14,3 \%)$ isolados foram susceptíveis a todos os antibióticos testados. Todos os isolados foram sensíveis a ciprofloxacina e gentamicina. Resultado semelhante foi encontrado em manipuladores no Rio Grande do Sul onde todos os isolados foram sensíveis a gentamicina ${ }^{12}$.

A resistência à penicilina foi a de maior frequência e foi de $74,2 \%$ entre os isolados obtidos dos manipuladores, 69,2\% para os de leite cru e $68,8 \%$ entre os obtidos a partir do queijo Minas Frescal. Resultados semelhantes para manipuladores $(70,0 \%)$ foram encontrados por Acco et al. ${ }^{12}$. No Chile, $100 \%$ das cepas isoladas de manipuladores foram resistentes à penicilina ${ }^{11}$. Em Porto Alegre, RS, 94,4\% das amostras obtidas dos manipuladores, $47,3 \%$ do leite cru e $50 \%$ do produto final foram resistentes à penicilina ${ }^{9}$. Lange et al..$^{35}$ observaram $43,9 \%$ de $S$. aureus isolados de leite cru resistentes à penicilina no Brasil e Calvinho et al (2002) ${ }^{13}$, em 47.6\% deste produto, na Argentina.

A utilização do antibiograma permitiu a observação de 18 (23,4\%) dos isolados, resistentes a mais de um antibiótico. Atualmente grande importância tem sido dada à multi-resistência antibiótica das bactérias, por sua correlação com virulência 
como os $S$. aureus meticilina-resistentes $(\text { MRSA })^{30}$. Neste estudo, quatro $(5,2 \%)$ isolados (três dos manipuladores e um de queijo) apresentaram padrão de MRSA. A disseminação de microrganismos resistentes, entre eles MRSA por alimentos e/ou manipuladores de alimentos deve ser considerada preocupante e evitada na cadeia de produção.

De acordo com os padrões de susceptibilidade originados pelo antibiograma, foi possível classificar os 77 isolados em doze perfis diferentes $(\mathrm{A}-\mathrm{L})$, como mostrado na tabela 3. Os S. aureus isolados de manipuladores foram classificados em nove diferentes perfis (A, B, C, D, F, I ,J, K e L), enquanto o leite cru em oito perfis (A, B, C, $\mathrm{D}, \mathrm{E}, \mathrm{F}, \mathrm{H}$ e I) e os isolados de queijo em oito perfis diferentes (A, B, C, D, E, F, G, e $J$ ), sugerindo diferentes origens de contaminação do produto final. De acordo com a tabela 3 , houve predominância de isolados com fenótipos A, B, C e D. Ainda na tabela 3, verifica-se que das 20 cepas de $S$. aureus obtidas a partir do queijo, cinco (25\%) possuem o fenótipo C.

Nossos resultados mostraram cepas indistinguíveis pelo antibiograma, colonizando manipuladores, leite e queijo, não permitindo a identificação da fonte destas bactérias para o produto final. Somente as amostras 3Q (perfil E) e 2Q (perfil J) apresentaram perfis correspondentes a somente uma das fontes prováveis, leite (13L, 14L') e manipulador (16M1), respectivamente.
Nos últimos anos, vários métodos de tipagem bacteriana têm sido usados para comparar cepas e identificar mecanismos de transmissão e fontes de contaminação para várias bactérias patogênicas ${ }^{24}$. Entre esses métodos, o antibiograma tem sido usado por ser de fácil execução, acessível, permitir controle de qualidade rigoroso e de baixo custo $^{25}$. Além disso permite o conhecimento acerca da resistência microbiana das bactérias testadas. Entretanto, o antibiograma apresenta limitações, como baixa sensibilidade, o que pode levar à determinação de pontos de controle inadequados ou identificação equivocada de fontes de contaminação ${ }^{9,14}$.

O elevado percentual de amostras de queijo Minas Frescal apresentando $S$. aureus, inclusive com contagens superiores à permitida pela legislação brasileira é bastante preocupante, pelo risco potencial de produção de enterotoxinas e conseqüente intoxicação alimentar. Além disso, cepas de $S$. aureus resistentes a antimicrobianos estão presentes em todas as fontes testadas e portanto, comprometendo a segurança da cadeia de produção do queijo, representando risco ao consumidor, inclusive com detecção de MRSA. Apesar de não ter sido eficiente em proceder a tipagem das cepas isoladas, o antibiograma forneceu informações importantes quanto à susceptibilidade das cepas e quanto ao perigo potencial de disseminação de cepas resistentes. Mais uma vez evidenciam-se falhas na produção deste alimento que continua sendo ainda um produto de baixa qualidade microbiológica, prejudicando a indústria nacional.

\section{Antibiogram as Instrument of Phenotypic Typing of Staphylococcus aureus Isolated from Personnel, Raw Milk and Minas Frescal Cheese in a Dairy Plant of Goiás, Brazil}

\section{Abstract}

During a year (March/2004 to February/2005) 140 samples of raw mill, dairy staff, and Minas Frescal cheese were analyzed for the presence of $S$. aureus in a dairy plant in Goiás, Brazil. Strains were isolated from $75 \%$ of the personnel investigated resulting in 31 (33.7\%) isolates from 92 collected samples (46 from hands and 46 from nasopharynx). The 24 raw milk samples resulted in $18(75.0 \%)$ positive samples and 26 isolates, with mean number: $1.1 \times 10^{5} \mathrm{CFU} / \mathrm{ml}$. Among 24 cheese samples $17(70.8 \%)$ were positive resulting in 20 isolates, with mean
Key-words:

Cheese.

Personnel. Antibiotic susceptibility. Staphylococcus aureus. 
number: $3.8 \times 104 \mathrm{CFU} / \mathrm{g}$. Thirteen samples (54.2\%) were above the limit established by the brazilian legislation $\left(10^{3} \mathrm{CFU} / \mathrm{g}\right)$. The differentiation of strains was performed by antimicrobial susceptibility test. All isolates were susceptible to ciprofloxacin and gentamicin. Resistance was observed in five $(6.5 \%)$ isolates for erythromycin, 19 $(24.7 \%)$ for tetracycline, one $(1.3 \%)$ for vancomycin, four $(5.2 \%)$ for oxacillin and $53(68.8 \%)$ isolates for penicillin. Eleven isolates (14.3\%) were susceptible to all antibiotics tested and $18(23.4 \%)$ were resistant to more than one antibiotic. The antibiotic susceptibility allowed the discrimination of isolates in 12 different profiles (A-L), but the phenotypic typing failed to determine the source of bacteria for the cheese.

\section{Referências}

1 ALMEIDA FILHO, E. S.; NADER FILHO, A. Ocorrência de Staphylococcus aureus em queijo tipo "frescal". Revista de Saúde Pública, v. 34, n. 6, p.578580, 2000.

2 DE BUYSER, M. L. et al. Implication of milk and milk products in food-borne diseases in France and in different industrialised countries. International Journal of Food Microbiology, v. 67, p. 1-17, 2001.

3 NAGASE, N. et al. Characterisation of Staphylococcus aureus strains isolated from bovine mastitis in Japan. Journal of Veterinary Medicine Science, v. 64, n. 12, p. 1169-1172, 2002.

4 VAUTOR, E. et al. Genotyping of Staphylococcus aureus isolated from various sites on farms with dairy sheep using pulsed-field gel electrophoresis. Veterinary Microbiology, v. 96, n. 1, p. 69-79, 2003.

5 AL BUSTAN, M. A.; UDO, E. E.; CHUGH, T. D. Nasal carriage of enterotoxin-producing Staphylococcus aureus among restaurant workers in Kuwait City. Epidemiology and Infection, v. 116, n. 3, p. 319-322, 1996.

6 SOTO, A. et al. Prevalence of Staphylococcus aureus among food handlers from a metropolitan university in Chile. Revista Médica de Chile, v. 124, n. 9, p. 1142-1146, 1996.

7 SOARES, M. J. S. et al. Enterotoxin production by Staphylococcus aureus clones and detection of brazilian epidemic MRSA clone (III::B:A) among isolates from food handlers. Journal of Medical Microbiology, v. 46, p. 214-221, 1997.

8 SUZUKI, Y.; SAITO, M.; ISHIKAWA, N. Restriction fragment length polymorphisms analysis by pulsedfield gel electrophoresis for discrimination of Staphylococcus aureus isolates from foodborne outbreaks. International Journal of Food Microbiology, v. 46 , p. $271-274,1999$.

9 TONDO, E. C. et al. Assessing and analysing contamination of a dairy products processing plant by Staphylococcus aureus using antibiotic resistance and
PFGE. Canadian Journal of Microbiology, v. 46, p. 1108-1114, 2000.

10 HATAKKA, M. et al. Genotypes and enterotoxicity of Staphylococcus aureus isolated from the hands and nasal cavities of flight-catering employees. Journal of Food Protection, v. 63, n. 11, p. 1487-1491, 2004.

11 FIGUEROA, G. G. et al. Carriage of enterotoxigenic Staphylococcus aureus in food handlers. Revista Médica de Chile, v. 130, n. 8, p. 859-864, 2002.

12 ACCO, M. et al. Identification of multiple strains of Staphylococcus aureus colonizing nasal mucosa of food handlers. Food Microbiology, v. 20, p. 489-493, 2003.

13 AYCIÇEK, H. et al. Assesment of the bacterial contamination on hands of hospital food handlers. Food Control, v. 15, p. 253-259, 2004.

14 AARESTRUP, F. M.; WEGENER, H. C.; ROSDAHL, V. T. Evaluation of phenotypic and genotypic methods for epidemiological typing of Staphylococcus aureus isolates from bovine mastitis in Denmark. Veterinary Microbiology, v. 45, p. 139, 1995.

15 DE OLIVEIRA, A. P. et al. Antimicrobial susceptibility of Staphylococcus aureus isolated from bovine mastitis in Europe and the United States. Journal of Dairy Science, v. 83, p. 855-862, 2000.

16 AKINEDEN, Ö. et al. Toxin genes and other characteristics of Staphylococcus aureus isolates from milk of cows with mastitis. Clinical and Diagnostic Laboratory Immunology, v. 8, p. 959-964, 2001.

17 VINTOV, J. et al. Phage types and antimicrobial resistance among Danish bovine Staphylococcus aureus isolates since the 1950s. Veterinary Microbiology, v. 97, n. 1-2, p. 63-72, 2003.

18 LANGE, C. et al. Molecular subtyping of Staphylococcus aureus isolates from cases of bovine mastitis in Brazil. Veterinary Microbiology, v. 67, p. 127-141, 1999.

19 GOÑI, P. et al. Antibiotic resistance and epidemiological typing of Staphylococcus aureus strains from ovine and rabbit mastitis. International Journal of Antimicrobial Agents, v. 23, n. 3, p. 268-272, 2004. 
20 SCHOCKEN-ITURRINO, R. P.; NADER FILHO, A. $\mathrm{N}$. Ação de antibióticos e quimioterápicos sobre alguns agentes bacterianos sobre alguns agentes bacterianos de mastite bovina. Revista do Instituto de Laticínios Cândido Tostes, v. 39, n. 233, p. 7-12, 1984.

21 BALABAN, N.; RASOOLY A. Staphylococcal enterotoxins. International Journal of Food Microbiology, v. 61, n. 1, p. 1-10, 2000.

22 ARAÚJO, V. S.; PAGLIARES, V. A.; QUEIROZ, M. L.; FREITAS-ALMEIDA, A. C. Occurrence of Staphylococcus and enteropathogens in soft cheese commercialized in the city of Rio de Janeiro, Brazil. Journal of Applied Microbiology, v. 92, n. 6, p. 1172 1177, 2002.

23 LOGuercio, A. P.; ALEIXO, J. A. G. Microbiologia de queijo tipo Minas frescal produzido artesanalmente. Ciência Rural, v. 31, n. 6, p. 10631067, 2001.

24 ZADOKS, R. N. et al. Comparison of Staphylococcus aureus isolates from bovine and human skin, milking, equipment, and bovine milk by phage typing, pulsedfield gel electrophoresis, and binary typing. Journal of Clinical Microbiology, v. 40, n. 11, p. 3894-3902, 2002.

25 ARBEIT, R. D. Laboratory procedures for the epidemiologic analysis of microrganisms. In MURRAY, P. R.; BARON, E. J.; PFALLER M. A.; TENOVER, F. C.; YOLKEN, R. H. Manual of clinical microbiology. 7ed. Washington, DC: American Society for Microbiology, 1999. p. 116-137.

26 MONTESINOS, I. et al. Epidemiologic genotyping of methicillin-resistant Staphylococcus aureus by pulsedfield gel electrophoresis at a University Hospital and comparison with antibiotyping and protein $\mathrm{A}$ and coagulase gene polymorphisms. Journal of Clinical Microbiology, v. 40, n. 6, p. 2119-2125, 2002.

27 LANCETTE, G. A.; TATINI, S. R. Staphylococcus aureus. In: VANDERZANT, C.; SPLITTSTOESSER, D. F. Compendium of methods for the microbiological examination of foods. 3.ed. Washington: American Public Health Association (APHA), 1992. p. 533-559.

28 KLOOS, W. E.; BANNERMAN, T. L. Staphylococcus and Micrococcus. In: MURRAY, P. R.; BARON, E. J. PFALLER, M. A.; TENOVER, F. C.; YOLKEN, R. H. Manual of clinical microbiology. 7ed. Washington: American Society for Microbiology. 1999. p. 264-282.

29 NATIONAL COMMITTEE FOR CLINICAL LABORATORY STANDARDS. Performance standards for antimicrobial disk susceptibility tests. Approved Standards. M2-A8. $8^{\text {th }}$ ed. Wayne, PA: NCCLS, 2003. $31 \mathrm{p}$.

30 KUMARI, D. N. et al. Comparison and application of ribosome spacer DNA amplicon polymorphisms and pulsed-field gel electrophoresis for differentiation of methicillin-resistant Staphylococcus aureus strains.
Journal of Clinical Microbiology, v. 35, n. 4, p. 881885, 1997.

31 ADESIYUN, A. A.; WEBB, L. A.; ROMAIN, H. T. Prevalence and characteristics of Staphylococcus aureus strains isolates from bulk and composite milk and cattle handlers. Journal of Food Protection, v. 61, p. 629632, 1998.

32 BUZZOLA, F. R. et al. Genotypic analysis of Staphylococcus aureus from milk of dairy cows with mastitis in Argentina. Epidemiology and Infection, $v$. 126, p. 445-452, 2001.

33 BRASIL. Ministério da Saúde. Agência Nacional de Vigilância Sanitária. Resolução-RDC $n^{\circ} 12$, de 2 de janeiro de 2001 - DOU de 10/01/2001. Regulamento técnico sobre padrões microbiológicos para alimentos, 2001.

34 REIBNITZ, M. G.; TAVARES, L. B.; GARCIA, J. A. Presence of fecal coliforms, Escherichia coli and DNAseand coagulase-positive Staphylococcus aureus, in "colonial" cheese sold in the city of Blumenau, Estado de Santa Catarina, Brazil. Revista Argentina de Microbiologia, v. 30, n. 1, p. 8-12, 1998.

35 CALVINHO, L. F. et al. Antimicrobial sensitivity of coagulase-positive staphylococcal strains isolated from bovine mastitis in the central dairy cathment area of Argentina. Revista Argentina de Microbiologia, v. 34 n. 3, p. 171-175, 2002

36 KLUYTMANS, J.; BELKUM, A.; VERBRUGH, $\mathrm{H}$ Nasal carriage of Staphylococcus aureus: epidemiology, underlying mechanisms, and associated risks. Clinical Microbiology Reviews, v. 10, p. 505-520, 1997.

37 VANDENBERGH, M. F. Q. et al. Follow-up of Staphylococcus aureus nasal carriage after 8 years: redefining the persistent carrier state. Journal of Clinical Microbiology, v. 37, n. 10, p. 3133-3140, 1999. 